\title{
On the Darboux-Nijenhuis Variables for the Open Toda Lattice ${ }^{\star}$
}

Yuriy A. GRIGORYEV and Andrey V. TSIGANOV

St.Petersburg State University, St.Petersburg, Russia

E-mail:com974@mail.ru,tsiganov@mph.phys.spbu.ru

Received November 17, 2006; Published online December 30, 2006

Original article is available at http://www.emis.de/journals/SIGMA/2006/Paper097/

\begin{abstract}
We discuss two known constructions proposed by Moser and by Sklyanin of the Darboux-Nijenhuis coordinates for the open Toda lattice.
\end{abstract}

Key words: bi-Hamiltonian systems; Toda lattice

2000 Mathematics Subject Classification: 37K10

\section{Introduction}

A bi-Hamiltonian manifold $M$ is a smooth manifold endowed with two compatible bi-vectors $P, P^{\prime}$ such that

$$
[P, P]=\left[P, P^{\prime}\right]=\left[P^{\prime}, P^{\prime}\right]=0,
$$

where $[\cdot, \cdot]$ is the Schouten bracket. The bi-vectors $P, P^{\prime}$ determine a pair of compatible Poisson brackets on $M$, for instance

$$
\{f(z), g(z)\}=\langle d f, P d g\rangle=\sum_{i, j}^{\operatorname{dim} M} P^{i j}(z) \frac{\partial f(z)}{\partial z_{i}} \frac{\partial g(z)}{\partial z_{j}}
$$

and similar brackets $\{\cdot, \cdot\}^{\prime}$ to $P^{\prime}$.

Dynamical systems on $M$ having enough functionally independent integrals of motion $H_{1}, \ldots$, $H_{n}$ in involution with respect to both Poisson brackets

$$
\left\{H_{i}, H_{j}\right\}=\left\{H_{i}, H_{j}\right\}^{\prime}=0 .
$$

will be called bi-integrable systems.

A sufficient condition in order that integrals of motion $H_{1}, \ldots, H_{n}$ be in bi-involution is that the corresponding vector fields $X_{H_{i}}$ are bi-Hamiltonian vector fields [13, 17], which form a so-called anchored Lenard-Magri sequence

$$
P d H_{1}=0, \quad X_{H_{i}}=P d H_{i}=P^{\prime} d H_{i-1}, \quad P^{\prime} d H_{n}=0 .
$$

The class of manifolds we will consider are particular bi-Hamiltonian manifolds, to be termed $\omega N$-manifolds, where one of the two Poisson bi-vectors is nondegenerate (say $P$ ) and thus defines a symplectic form $\omega=P^{-1}$ and, together with the other one, a recursion operator $[3,4]$

$$
N=P^{\prime} P^{-1}
$$

*This paper is a contribution to the Vadim Kuznetsov Memorial Issue "Integrable Systems and Related Topics". The full collection is available at http://www.emis.de/journals/SIGMA/kuznetsov.html 
and its dual $N^{*}=P^{-1} P^{\prime}$. Operator $N$ is called a Nijenhuis operator $[13,17]$ or hereditary operator $[10,8]$.

One of the main property of the recursion operator $N$ is that bi-vectors $P=P^{(0)}$ and $P^{\prime}=P^{(1)}$ belong to a whole family of compatible Poisson tensors

$$
P^{(k)}=N^{k} P,
$$

which define a family of compatible Poisson brackets $\{\cdot, \cdot\}^{(k)}$ on the $\omega N$-manifold $M$.

Another useful property of $N$ is that normalized traces of the powers of $N$ are integrals of motion satisfying Lenard-Magri recurrent relations (1.3) [17]:

$$
H_{j}=\frac{1}{2 j} \operatorname{tr} N^{j}
$$

The class of coordinates, called Darboux-Nijenhuis coordinates, are canonical with respect to $\omega$ and diagonalize recursion operator $N$. According to [3, 4], the $n$-tuple $\left(H_{0}, \ldots, H_{n}\right)$ of Hamiltonians on $M$ (where $n=\frac{1}{2} \operatorname{dim} M$ ) is separable in Darboux-Nijenhuis coordinates if and only if they are in involution with respect to both Poisson brackets (1.2).

In this paper we compare two known families of the separated variables for the open Toda lattice $[18,19]$ with the corresponding Darboux-Nijenhuis coordinates.

\section{The separation of variables method}

In the separation of variables method variables we are looking for complete integral $S\left(q, t, \alpha_{1}, \ldots\right.$, $\alpha_{n}$ ) of the Hamilton-Jacobi equation

$$
\frac{\partial S}{\partial t}+H\left(q, \frac{\partial S}{\partial q}, t\right)=0, \quad \operatorname{det}\left\|\frac{\partial^{2} S}{\partial q_{i} \partial \alpha_{j}}\right\| \neq 0
$$

where $q=\left(q_{1}, \ldots, q_{n}\right)$, in the additive form

$$
S\left(q, t, \alpha_{1}, \ldots, \alpha_{n}\right)=-H t+\sum_{i=1}^{n} S_{i}\left(q_{i}, \alpha_{1}, \ldots, \alpha_{n}\right) .
$$

Here the $i$-th component $S_{i}$ depends only on the $i$-th coordinate $q_{i}$ and $n$ parameters $\alpha_{1}, \ldots, \alpha_{n}$ which are the values of integrals of motion. In such a case $H$ is said to be separable and coordinates $q$ are said to be separated coordinates for $H$, in order to stress that the possibility to find an additive complete integral of (2.2) depends on the choice of the coordinates.

For any complete integral of the equation (2.1) solutions $q_{i}=q_{i}(t, \alpha, \beta)$ and $p_{i}=p_{i}(t, \alpha, \beta)$ of the Hamilton equations of motion are found from the Jacobi equations

$$
\beta_{i}=-\frac{\partial S}{\partial \alpha_{i}}, \quad p_{i}=\frac{\partial S}{\partial q_{i}}, \quad i=1, \ldots, n .
$$

In the separation of variables method the each second Jacobi equation

$$
p_{i}=\frac{\partial}{\partial q_{i}} S_{i}\left(q_{i}, \alpha_{1}, \ldots, \alpha_{n}\right),
$$

contains the pair of the Darboux coordinates $p_{i}$ and $q_{i}$ only. These equations and their more symmetric form

$$
\Phi_{i}\left(q_{i}, p_{i}, \alpha_{1}, \ldots, \alpha_{n}\right)=0, \quad \operatorname{det}\left\|\frac{\partial \Phi_{j}}{\partial \alpha_{k}}\right\| \neq 0
$$

are called the separated equations. 
The separated variables $(p, q)$ are defined up to canonical transformations $p_{i} \rightarrow f_{i}\left(p_{i}, q_{i}\right)$ and $q_{i} \rightarrow g_{i}\left(p_{i}, q_{i}\right)$ and integrals of motion $H_{j}=\alpha_{j}$ can be always replaced with $\tilde{H}_{j}=\phi_{j}\left(H_{1}, \ldots, H_{n}\right)$. Such transformations change the form of the separated equations (2.5).

For example, we can use canonical transformation to the action-angle variables

$$
I_{j}=\frac{1}{2 \pi} \oint p_{j} d q_{j}=\frac{1}{2 \pi} \oint \frac{\partial S_{j}\left(q_{j}, \alpha_{1}, \ldots, \alpha_{n}\right)}{\partial q_{j}} d q_{j}, \quad w_{j}=\frac{\partial W}{\partial I_{j}} .
$$

Here $W=\sum_{i=1}^{n} S_{i}\left(q_{i}, \alpha_{1}, \ldots, \alpha_{n}\right)$ is Hamilton's characteristic function. The action-angle variables $I, w$ are the special separated variables which allow us to linearize equations of motion

$$
\dot{I}_{j}=0, \quad \dot{w}_{j}=F_{j}\left(I_{1}, \ldots, I_{n}\right), \quad j=1, \ldots, n .
$$

However, the action-angle variables are not always convenient, for instance for the quantum integrable system.

Example 1. Consider a two-particle open Toda chain with the following integrals of motion

$$
H_{1}=p_{1}+p_{2}, \quad H_{2}=\frac{p_{1}^{2}+p_{2}^{2}}{2}+e^{q_{1}-q_{2}} .
$$

Variables

$$
v_{1,2}=\frac{1}{\sqrt{2}}\left(q_{1} \pm q_{2}\right), \quad u_{1,2}=\frac{1}{\sqrt{2}}\left(p_{1} \pm p_{2}\right)
$$

are separated variables, because substituting these variables in the definition of integrals of motion we obtain desired separated equations (2.5)

$$
\Phi_{1}\left(v_{1}, u_{1}, \alpha\right)=\sqrt{2} u_{1}-H_{1}=0, \quad \Phi_{2}\left(v_{2}, u_{2}, \alpha\right)=e^{\sqrt{2} v_{2}}+\frac{u_{2}^{2}}{2}-H_{2}+\frac{H_{1}^{2}}{4}=0 .
$$

Provided $H_{i}=\alpha_{i}$ and $u_{i}=\frac{\partial S_{i}}{\partial v_{i}}$, these separated equations

$$
\frac{\partial S_{1}}{\partial v_{1}}=\frac{\alpha_{1}}{\sqrt{2}}, \quad \frac{1}{2}\left(\frac{\partial S_{2}}{\partial v_{2}}\right)^{2}+e^{\sqrt{2} v_{2}}-\alpha_{2}+\frac{\alpha_{1}^{2}}{4}=0
$$

can easily be integrated by quadratures

$$
\begin{aligned}
& S_{1}=\frac{\alpha_{1} v_{1}}{\sqrt{2}} \\
& S_{2}=\mp \sqrt{4 \alpha_{2}-\alpha_{1}^{2}-4 e^{\sqrt{2} v_{2}}} \pm \sqrt{4 \alpha_{2}-\alpha_{1}^{2}} \operatorname{arctanh}\left(\frac{\sqrt{4 \alpha_{2}-\alpha_{1}^{2}-4 e^{\sqrt{2} v_{2}}}}{\sqrt{4 \alpha_{2}-\alpha_{1}^{2}}}\right) .
\end{aligned}
$$

Then we have to substitute the corresponding Hamilton principal function $S=-\alpha_{2} t+S_{1}+S_{2}$ in the Jacobi equations

$$
\begin{aligned}
& \beta_{1}=-\frac{\partial S}{\partial \alpha_{1}}=-\frac{v_{1}}{\sqrt{2}}+\frac{\alpha_{1}}{\sqrt{4 \alpha_{2}-\alpha_{1}^{2}}} \operatorname{arctanh}\left(\frac{\sqrt{4 \alpha_{2}-\alpha_{1}^{2}-4 e^{\sqrt{2} v_{2}}}}{\sqrt{4 \alpha_{2}-\alpha_{1}^{2}}}\right), \\
& \beta_{2}=-\frac{\partial S}{\partial \alpha_{2}}=t-\frac{2}{\sqrt{4 \alpha_{2}-\alpha_{1}^{2}}} \operatorname{arctanh}\left(\frac{\sqrt{4 \alpha_{2}-\alpha_{1}^{2}-4 e^{\sqrt{2} v_{2}}}}{\sqrt{4 \alpha_{2}-\alpha_{1}^{2}}}\right),
\end{aligned}
$$


and solve the obtained equations with respect to $v_{1,2}$

$$
\begin{aligned}
& v_{1}=\frac{\alpha_{1}\left(t-\beta_{2}\right)}{\sqrt{2}}+\sqrt{2} \beta_{1} \\
& v_{2}=\sqrt{2} \ln \left(\alpha_{2}-\frac{\alpha_{1}^{2}}{4}\right)-2 \sqrt{2} \ln \left(\cosh \left(\sqrt{\alpha_{2}-\frac{\alpha_{1}^{2}}{4}}\left(t-\beta_{2}\right)\right)\right) .
\end{aligned}
$$

Using these solutions and canonical transformation (2.8) we obtain closed equations for trajectories of motion in the original variables

$$
q_{1,2}=\frac{\alpha_{1}\left(t-\beta_{2}\right)}{2} \pm \frac{1}{2} \ln \left(\alpha_{2}-\frac{\alpha_{1}^{2}}{4}\right) \mp \ln \left(\cosh \left(\sqrt{\alpha_{2}-\frac{\alpha_{1}^{2}}{4}}\left(t-\beta_{2}\right)\right)\right)-\beta_{1} .
$$

In similar way we can get $p_{1,2}(t)$ from the second Jacobi equations (2.4).

Parameters $\beta_{1,2}$ may be excluded by the shifts $t \rightarrow t+\beta_{2}$ and $q_{i} \rightarrow q_{i}+\beta_{1}$ and, thus, the solutions $q_{1,2}(t)$ depend on two values of integrals of motion $\alpha_{1,2}$ only.

Now from (2.6) one can easily find the action variables

$$
I_{1}=\alpha_{1}=\sqrt{2} u_{1}, \quad I_{2}=4 \alpha_{2}-\alpha_{1}^{2}=2 u_{2}^{2}+4 e^{\sqrt{2} v_{2}}
$$

and the angle variables

$$
w_{1}=\frac{\partial S}{\partial I_{1}}=\frac{v_{1}}{\sqrt{2}}, \quad w_{2}=\frac{\partial S}{\partial I_{1}}=-\frac{1}{2 \sqrt{2}} \frac{\operatorname{arctanh}\left(\frac{u_{2}}{\sqrt{u_{2}^{2}+2 e^{\sqrt{2} v_{2}}}}\right)}{\sqrt{u_{2}^{2}+2 e^{\sqrt{2} v_{2}}}} .
$$

In these coordinates symplectic form has a standard form

$$
\omega=\sum_{j=1}^{2} d p_{j} \wedge d q_{j}=\sum_{j=1}^{2} d I_{j} \wedge d w_{j}
$$

and equations of motion are linearized

$$
\dot{I}_{j}=0, \quad \dot{w}_{1}=\frac{\partial H}{\partial I_{1}}=\frac{I_{1}}{2}, \quad \dot{w}_{2}=\frac{\partial H}{\partial I_{2}}=\frac{1}{4} .
$$

Using solutions of these equations and inverted canonical transformation $I, w \rightarrow p, q$ one can easily derive equations for the trajectories of motion (2.10).

Separated variables $v_{1,2}$ and $u_{1,2}$ can also be used in quantum mechanics, for example, to find the spectrum of Hamiltonian of a periodical two-particle Toda chain [14].

\subsection{Darboux-Nijenhuis coordinates}

In this section we will describe a class of canonical coordinates on $\omega N$-manifolds, called DarbouxNijenhuis coordinates. They will play the important role of variables of separation for (suitable) systems on $\omega N$-manifolds.

By definition a set of local coordinates $\left(x_{i}, y_{i}\right)$ on an $\omega N$-manifold is called a set of DarbouxNijenhuis coordinates if they are canonical with respect to the symplectic form

$$
\omega=P^{-1}=\sum_{i=1}^{n} d y_{i} \wedge d x_{i}
$$


and put the recursion operator $N$ in diagonal form,

$$
N=\sum_{i=1}^{n} \lambda_{i}\left(\frac{\partial}{\partial x_{i}} \otimes d x_{i}+\frac{\partial}{\partial y_{i}} \otimes d y_{i}\right) .
$$

This means that the only nonzero Poisson brackets are

$$
\left\{x_{i}, y_{j}\right\}=\delta_{i j}, \quad\left\{x_{i}, y_{j}\right\}^{\prime}=\lambda_{i} \delta_{i j}
$$

The distinguishing property of the pairs of Darboux-Nijenhuis coordinates $\left(x_{i}, y_{i}\right)$ is that their differentials span an eigenspace of $N^{*}$, that is, satisfy the equations

$$
N^{*} d x_{i}=\lambda_{i} d x_{i}, \quad N^{*} d y_{i}=\lambda_{i} d y_{i}
$$

As a consequence of the compatibility between $P$ and $P^{\prime}$, the Nijenhuis torsion of $N$ vanishes

$$
T_{N}(X, Y)=[N X, N Y]-N([N X, Y]+[X, N Y]-N[X, Y])=0,
$$

here $X, Y$ are arbitrary vector fields on $M$.

According to the Frölicher-Nijenhuis theory [9], condition (2.16) implies that the distribution of the eigenvectors of $N$ is integrable. In application to Darboux-Nijenhuis coordinates it means that it is possible to find by quadratures $2 n$ functions $\left(x_{i}, y_{i}\right)$ directly from the equations (2.15) [11, 16]. Hence, the Frölicher-Nijenhuis theory allows one to construct the DarbouxNijenhuis variables as solutions of the equations (2.15) or their equivalent (2.14).

However, for any function $f\left(x_{i}, y_{i}\right)$ equation

$$
N^{*} d f\left(x_{i}, y_{i}\right)=\lambda_{i} d f\left(x_{i}, y_{i}\right)
$$

is also satisfied. Hence, any pair of Darboux-Nijenhuis coordinates is defined up to arbitrary canonical transformations. So in the general case equations (2.15) have infinitely many solutions and, thus, it is impossible to create a common effective algorithm to solve these equations.

This problem can be partially solved. As a consequence of the vanishing of the Nijenhuis torsion of $N$ the eigenvalues $\lambda_{i}$ always satisfy (2.15)

$$
N^{*} d \lambda_{i}=\lambda_{i} d \lambda_{i}
$$

The eigenvalues $\lambda_{i}$ and their conjugated variables $\mu_{i}$ are the special Darboux-Nijenhuis coordinates [4], which are distinguished because $\lambda_{i}$ are simply the roots of the minimal characteristic polynomial of $N$

$$
\Delta_{N}(\lambda)=(\operatorname{det}(N-\lambda \mathrm{I}))^{1 / 2}=\lambda^{n}-\left(c_{1} \lambda^{n-1}+\cdots+c_{n}\right)=\prod_{j=1}^{n}\left(\lambda-\lambda_{j}\right) .
$$

Here $c_{j}\left(\lambda_{1}, \ldots, \lambda_{n}\right)$ are elementary symmetric polynomials of power $j$ which are related with integrals of motion $H_{j}(1.6)$ by the Newton formulas.

The complimentary variables $\mu_{j}$ must be calculated as solutions of the overdetermined system of partial differential equations (2.15)

$$
N^{*} d \mu_{i}=\lambda_{i} d \mu_{i}, \quad\left\{\lambda_{i}, \mu_{j}\right\}=\delta_{i j}, \quad\left\{\mu_{i}, \mu_{j}\right\}=0,
$$

whose solutions $\mu_{j}$ are still determined up to infinitely many canonical transformations $\mu_{j} \rightarrow$ $\mu_{j}+f\left(\lambda_{j}\right)$

An effective algorithm for calculating Darboux-Nijenhuis variables has not been developed yet despite these variables play an important role in the method of separation of variables due to the following theorem. 
Theorem 1 ([3, 4]). If $M$ is a $2 n$-dimensional $\omega N$-manifold such that in the neighborhood of any point $z \in M$ recursion operator $N$ has $n$ different functionally independent eigenvalues and if $\left\{H_{1}, \ldots, H_{n}\right\}$ is a family of independent functions on $M$, then the following statements are equivalent:

1) functions $\left\{H_{1}, \ldots, H_{n}\right\}$ are in bi-involution (1.2);

2) the Lagrangian foliation $\mathcal{F}$ defined by $\left\{H_{1}, \ldots, H_{n}\right\}$ is separable in Darboux-Nijenhuis coordinates;

3) the distribution $\mathcal{D}$ tangent to the foliation defined by $\left\{H_{1}, \ldots, H_{n}\right\}$ is Lagrangian with respect to $\omega=P^{-1}$ and invariant with respect to $N$.

It easy to prove that for integrable after Liouville bi-Hamiltonian systems with the integrals of motion $H_{j}$ (1.6) recursion operator $N$ has exactly $n$ functionally independent eigenvalues, because $H_{j}=j^{-1} \sum_{i=1}^{n} \lambda_{i}^{j}$ and

$$
d H_{1} \wedge \cdots \wedge d H_{n}=\prod_{i \neq j}\left(\lambda_{i}-\lambda_{j}\right) d \lambda_{1} \wedge \cdots \wedge d \lambda_{n}
$$

For bi-Hamiltonian systems integrals of motion $H_{i}$ (1.6) define a distinguished bi-Lagrangian foliation, called principal foliation [4].

For generic bi-integrable systems invariance of the distribution $\mathcal{D}$ with respect to $N$ means that there is a control matrix $F$ with eigenvalues $\left(\lambda_{1}, \ldots, \lambda_{n}\right)$ such that

$$
N^{*} d H_{i}=\sum_{k=1}^{n} F_{i j} d H_{j}, \quad i=1, \ldots, n .
$$

In the Darboux-Nijenhuis coordinates equations (2.19) may be considered as the known LeviCivita criterion for separability [15], see [4].

In particular case for bi-Hamiltonian systems (1.3) the control matrix $F$ has the following form

$$
F=\left(\begin{array}{ccccc}
0 & 1 & 0 & \cdots & 0 \\
0 & 0 & 1 & \cdots & 0 \\
\vdots & & \cdots & 0 & 1 \\
c_{n} & c_{n+1} & \cdots & & c_{1}
\end{array}\right)
$$

Here $c_{k}$ are coefficients of the characteristic polynomial $\Delta_{N}(\lambda)(2.17)$ of the recursion operator $N$.

Example 2. According to $[2,6]$, for a two-particle open Toda chain recursion operator reads as

$$
N=\left(\begin{array}{cccc}
p_{1} & 0 & 0 & 1 \\
0 & p_{2} & -1 & 0 \\
0 & -e^{q_{1}-q_{2}} & p_{1} & 0 \\
e^{q_{1}-q_{2}} & 0 & 0 & p_{2}
\end{array}\right)
$$

Integrals of motion (2.7) are reproduced by $N(1.6)$. Therefore eigenvalues of $N$

$$
\lambda_{1,2}=\frac{p_{1}+p_{2}}{2}+\frac{\sqrt{p_{1}^{2}+p_{2}^{2}-2 p_{1} p_{2}+4 \exp \left(q_{1}-q_{2}\right)}}{2} .
$$

are a half of the special Darboux-Nijenhuis coordinates for the open Toda lattice. Of course, these coordinates are the action variables $\dot{\lambda}_{i}=0$. 
The complimentary variables $\mu_{1,2}$ must be calculated as solutions of the overdetermined system of partial differential equations (2.18). However even in this simple case we could not directly solve these thirteen PDEs (2.18) for the two unknown functions $\mu_{i}\left(q_{1}, q_{2}, p_{1}, p_{2}\right)$.

Nevertheless we can find variables $\mu_{i}$ by using the action-angle variables $(2.11),(2.12)$ obtained before. Since

$$
\lambda_{i}=\frac{I_{1} \pm \sqrt{I_{2}}}{2}
$$

the second half of the special Darboux-Nijenhuis coordinates reads as

$$
\mu_{i}=w_{1} \pm 2 \sqrt{I_{2}} w_{2}=\frac{q_{1}-q_{2}}{2} \mp \operatorname{arctanh}\left(\frac{p_{1}-p_{2}}{\sqrt{\left(p_{1}-p_{2}\right)^{2}+4 \exp \left(q_{1}-q_{2}\right)}}\right) .
$$

By definition action variables $\lambda_{i}$ are roots of the minimal characteristic polynomial

$$
\Delta_{N}(\lambda)=\lambda^{2}-\left(p_{1}+p_{2}\right) \lambda+p_{1} p_{2}-e^{q_{1}-q_{2}},
$$

whereas angle variables $\mu_{i}$ can be defined in the following way:

$$
\mu_{i}=\ln B\left(\lambda_{i}\right), \quad B(\lambda)=-e^{q_{2}}\left(\lambda-p_{1}\right) .
$$

Summing up, to build the special Darboux-Nijenhuis variables we de-facto had to use another set of separated variables $v_{i}, u_{i}$ which are not Darboux-Nijenhuis variables.

In the next section we discuss how analogous variables $u$ and $v$ are related with the special Darboux-Nijenhuis variables for $n$-particle open Toda lattice.

\section{Open Toda lattice}

Let us consider open Toda associated with the root system of $\mathscr{A}_{n}$ type. The Hamilton function is equal to

$$
H=\frac{1}{2} \sum_{i=1}^{n} p_{i}^{2}+\sum_{i=1}^{n-1} e^{q_{i}-q_{i+1}}
$$

Here $p, q$ are Darboux coordinates on the manifold $M \simeq \mathbb{R}^{2 n}$

$$
\left\{q_{i}, p_{j}\right\}=\delta_{i j}, \quad\left\{p_{i}, p_{j}\right\}=\left\{q_{i}, q_{j}\right\}=0 .
$$

Bi-Hamiltonian structure of Toda chains was investigated both in terms of physical variables $(p, q)$ and in terms of so-called Flaschka variables [7]. We will use original physical variables $p$ and $q$, for which second Poisson tensor has the form $[2,6]$

$$
P^{\prime}=\sum_{i=1}^{n-1} e^{q_{i}-q_{i+1}} \frac{\partial}{\partial p_{i+1}} \wedge \frac{\partial}{\partial p_{i}}+\sum_{i=1}^{n} p_{i} \frac{\partial}{\partial q_{i}} \wedge \frac{\partial}{\partial p_{i}}+\sum_{i<j}^{n} \frac{\partial}{\partial q_{j}} \wedge \frac{\partial}{\partial q_{i}} .
$$

Throughout the rest of the paper $N$ is recursion operator $N=P^{\prime} P^{-1}$ for the open Toda lattice, where $P^{\prime}$ is given by (3.2) and $P$ is canonical tensor associated with canonical Poisson brackets (3.1) in $\mathbb{R}^{2 n}$. 


\subsection{The Moser method}

In this section we will give a brief review of a method for integrating equations of motion for an open Toda lattice proposed by Moser [18]. A contemporary algebraic-geometric review of the Moser method can be found in [25].

Let us start with a $n \times n$ Lax matrix $L$ with the components

$$
L_{j k}=p_{j} \delta_{j, k}+e^{\frac{q_{j}-q_{j+1}}{2}}\left(\delta_{j, k+1}+\delta_{j-1, k}\right) .
$$

Following [18] we define the Weyl function

$$
\mathcal{X}(\lambda)=(R(\lambda) \vec{\alpha}, \vec{\alpha}), \quad \vec{\alpha}=\left(0,0, \ldots, e^{q_{n} / 2}\right),
$$

where $R(\lambda)=(L-\lambda I)^{-1}$ is a resolvent of the Jacobi matrix (3.3) and $\vec{\alpha}$ is dynamical normalization of the corresponding Baker-Akhiezer function. It is known since the work of Stiltjies [22], the Weyl function plays the key role in reconstruction of the matrix $L$ from its spectral data and it is a ratio of the two monic polynomials

$$
\mathcal{X}(\lambda)=\frac{B(\lambda)}{A(\lambda)}
$$

where $B(\lambda)$ is a polynomial of degree $(n-1)$ and $A(\lambda)=\operatorname{det}(L-\lambda I)$ is a polynomial of degree $n$ with distinct roots $\lambda_{j}$

$$
A(\lambda)=\prod_{i=1}^{n}\left(\lambda-\lambda_{j}\right), \quad B(\lambda)=-e^{q_{n}}\left(\lambda^{n-1}+b_{2} \lambda^{n-2}+\cdots+b_{0}\right) .
$$

Proposition 1. For the open Toda lattice two Poisson brackets associated with tensors $P=P^{(0)}$ (1.5) and $P^{\prime}=P^{(1)}$ (3.2) form quadratic algebras for the polynomials $A(\lambda), B(\lambda)$ and for the Weyl function $\mathcal{X}(\lambda)$.

It is easy to prove that at $k=0,1$ these quadratic brackets are equal to

$$
\begin{aligned}
& \{A(\lambda), A(\mu)\}^{(k)}=\{B(\lambda), B(\mu)\}^{(k)}=0, \\
& \{A(\lambda), B(\mu)\}^{(k)}=\frac{\mu^{k} A(\lambda) B(\mu)-\lambda^{k} A(\mu) B(\lambda)}{\lambda-\mu}
\end{aligned}
$$

and

$$
\{\mathcal{X}(\lambda), \mathcal{X}(\mu)\}^{(k)}=\frac{(\mathcal{X}(\lambda)-\mathcal{X}(\mu))\left(\mu^{k} \mathcal{X}(\lambda)-\lambda^{k} \mathcal{X}(\mu)\right)}{\lambda-\mu} .
$$

At $k=0$ brackets (3.7) are the part of the Sklyanin brackets [19] and nontrivial expression in the right hand side of (3.7) is called a Bezoutian of polynomials $A$ and $B$ [12].

At $k=0$ brackets (3.8) give the Atiyah-Hitchin Poisson structure in the space of meromorphic $\operatorname{maps} \mathcal{X}(\lambda): \mathbb{C P}^{1} \rightarrow \mathbb{C P}^{1}[1]$.

Remark 1. According to [5] the Poisson brackets between polynomials $A(\lambda)$ and $B(\mu)$ associated with Poisson tensors $P^{(k)}(1.5)$ at $k=0,1, \ldots, n$ are equal to

$$
\begin{aligned}
\{A(\lambda), B(\mu)\}^{(k)} & =\frac{A(\lambda) B^{[k]}(\mu)-A(\mu) B^{[k]}(\lambda)}{\lambda-\mu} \\
& =\frac{\mu^{k} A(\lambda) B(\mu)-\lambda^{k} A(\mu) B(\lambda)}{\lambda-\mu}+A(\lambda) A(\mu) \frac{\left(\beta^{[k]}(\lambda)-\beta^{[k]}(\mu)\right)}{\lambda-\mu} .
\end{aligned}
$$


The right hand side of (3.9) contains either remainder

$$
B^{[k]}(\lambda)=\lambda^{k} B(\lambda) \bmod A(\lambda),
$$

either result of simple division in the space of polynomials

$$
\beta^{[k]}=\frac{\lambda^{k} B(\lambda)}{A(\lambda)}
$$

which is polynomial part of the Laurent decomposition of the ratio $\lambda^{k} B(\lambda) / A(\lambda)$. It is easy to see that $\beta^{[1]}=0$ and $\beta^{[1]}=-e^{q_{n}}$ and from (3.9) one gets (3.7).

In the Moser approach to the open Toda lattice [18] we can introduce the action-angle variables using the Weyl function (3.4). Namely, action coordinates are poles $\lambda_{i}$ (3.6) of the Weyl function $\mathcal{X}(\lambda)$, whereas angle variables are given by

$$
\mu_{j}=\ln B\left(\lambda_{j}\right) .
$$

It is easy to check that polynomial $A(\lambda)=\operatorname{det}(L-\lambda I)=\Delta_{N}(\lambda)$ is a minimal characteristic polynomial of the recursion operator $N$. Moreover, it follows from (3.7) that $\lambda_{i}$ and $\mu_{j}$ satisfy the necessary relations $(2.14)$

$$
\left\{\lambda_{i}, \mu_{j}\right\}=\delta_{i j}, \quad\left\{\lambda_{i}, \mu_{j}\right\}^{\prime}=\lambda_{i} \delta_{i j}
$$

We could avoid calculating of the second Poisson brackets between $A$ and $B$ by means of the equation

$$
N^{*} d \Delta_{N}(\lambda)=\lambda d \Delta_{N}(\lambda)+\Delta_{N}(\lambda) d c_{1},
$$

which can be obtained from the recurrent Lenard-Magri relations (1.3) rewritten in the form $N^{*} d H_{k}=d H_{k+1}$ and from the Newton formulas connecting $H_{k}$ with coefficients $c_{k}$ [4]. Combining this equation with a result of [23]

$$
B(\lambda)=-e^{q_{n}} \frac{\partial}{\partial c_{1}} \Delta_{N}(\lambda)
$$

one can easily prove that $B(\lambda)$ is a Stäckel function [4]

$$
N^{*} d B(\lambda)=\lambda d B(\lambda)+\Delta_{N}(\lambda) d e^{q_{n}}
$$

and, thus,

$$
N^{*} d \mu_{i}=\lambda_{i} d \mu_{i}
$$

Summing up, the Moser variables are the special Darboux-Nijenhuis variables for the open Toda lattice. The corresponding equations of motion have the form

$$
\left\{H_{i}, \lambda_{j}\right\}=\partial_{\tau_{i}} \lambda_{j}=0, \quad\left\{H_{i}, \mu_{j}\right\}=\partial_{\tau_{i}} \mu_{j}=\lambda_{j}^{i-1} .
$$

Evolution of the variables $\mu_{j}$ with respect to the times $\tau_{i}$ conjugated to the bi-Hamiltonian integrals of motion $H_{i}$ (1.6) is linear.

Brackets(3.8) for the Weyl function are invariant with respect to linear-fractional transformations

$$
\mathcal{X} \rightarrow \mathcal{X}^{\prime}=\frac{a \mathcal{X}+b}{c \mathcal{X}+d}
$$

and, therefore, we can introduce another family of the separated variables using the same Weyl function [25]. This second coordinate system is considered in the next section in framework of the classical $r$-matrix theory. 


\subsection{The Sklyanin method}

In this section we briefly discuss an application of the generic Sklyanin method [19, 20] to the open Toda lattice. Let us consider a $2 \times 2$ monodromy matrix

$$
T(\lambda)=\left(\begin{array}{cc}
A & B \\
C & D
\end{array}\right)(\lambda)=L_{1}(\lambda) \cdots L_{n-1}(\lambda) L_{n}(\lambda)
$$

where

$$
L_{i}=\left(\begin{array}{cc}
\lambda-p_{i} & -e^{q_{i}} \\
e^{-q_{i}} & 0
\end{array}\right) .
$$

Monodromy matrix $T(\lambda)$ (3.15) is the Lax matrix for periodic Toda lattice [19], whereas the Lax matrix for open Toda lattice is equal to

$$
T_{o}(\lambda)=K T(\lambda)=\left(\begin{array}{cc}
A & B \\
0 & 0
\end{array}\right)(\lambda), \quad K=\left(\begin{array}{ll}
1 & 0 \\
0 & 0
\end{array}\right) .
$$

The entries $A$ and $B$ of this $2 \times 2$ Lax matrix coincide with denominator and numerator of the Weyl function (3.5) respectively.

Proposition 2. The Poisson brackets between entries of the monodromy matrix $T(\lambda)(3.15)$ associated with Poisson tensors $P^{(k)}(1.5)$ at $k=0,1$ are equal to

$$
\begin{aligned}
\{\stackrel{1}{T}(\lambda), \stackrel{2}{T}(\mu)\}^{(k)}= & r_{12}(\lambda, \mu) \stackrel{2}{T}(\lambda) \stackrel{2}{T}(\mu)-\stackrel{1}{T}(\lambda) \stackrel{2}{T}(\mu) r_{21}(\lambda, \mu) \\
& +\stackrel{1}{T}(\lambda) s(\lambda, \mu) \stackrel{2}{T}(\mu)-\stackrel{2}{T}(\lambda) s(\lambda, \mu) \stackrel{1}{T}(\mu)
\end{aligned}
$$

where first r-matrix is equal to

$$
r_{12}(\lambda, \mu)=\frac{-1}{\lambda-\mu}\left(\begin{array}{cccc}
1 & 0 & 0 & 0 \\
0 & 1-\frac{\lambda^{k}+\mu^{k}}{2} & \mu^{k} & 0 \\
0 & \lambda^{k} & 1-\frac{\lambda^{k}+\mu^{k}}{2} & 0 \\
0 & 0 & 0 & 1
\end{array}\right), \quad r_{i j}(\lambda, \mu)=\Pi r_{j i}(\lambda, \mu) \Pi
$$

whereas the second matrix $s(\lambda, \mu)$ reads as

$$
s(\lambda, \mu)=\frac{-1}{\lambda-\mu}\left(\begin{array}{cccc}
0 & 0 & 0 & 0 \\
0 & \frac{\lambda^{k}-\mu^{k}}{2} & 0 & 0 \\
0 & 0 & \frac{\lambda^{k}-\mu^{k}}{2} & 0 \\
0 & 0 & 0 & 0
\end{array}\right)
$$

Here, $\stackrel{1}{T}(\lambda)=T(\lambda) \otimes I, \stackrel{2}{T}(\mu)=I \otimes T(\mu)$ and $\Pi$ is a permutation matrix in auxiliary space, i.e. $\Pi X \otimes Y=Y \otimes X \Pi$ for arbitrary matrices $X, Y$

At $k=0$ first matrix $r(\lambda, \mu)=-(\lambda-\mu)^{-1} \Pi$ is the standard rational $r$-matrix, while the second matrix $s(\lambda, \mu)$ is equal to zero. In this case quadratic brackets (3.16) are the Sklyanin bracket [19].

Proposition 3. In generic case the Poisson brackets $\{\cdot, \cdot\}^{(k)}$ associated with Poisson tensors $P^{(k)}(1.5)$ at $k=0,1, \ldots, n$ are equal to:

$$
\{A(\lambda), A(\mu)\}^{(k)}=\{B(\lambda), B(\mu)\}^{(k)}=\{C(\lambda), C(\mu)\}^{(k)}=\{D(\lambda), D(\mu)\}^{(k)}=0,
$$




$$
\begin{aligned}
& \{A(\lambda), B(\mu)\}^{(k)}=\frac{\mu^{k} A(\lambda) B(\mu)-\lambda^{k} A(\mu) B(\lambda)}{\lambda-\mu}+A(\lambda) A(\mu) \frac{\left(\beta^{[k]}(\lambda)-\beta^{[k]}(\mu)\right)}{\lambda-\mu}, \\
& \{A(\lambda), C(\mu)\}^{(k)}=-\frac{\mu^{k} A(\lambda) C(\mu)-\lambda^{k} A(\mu) C(\lambda)}{\lambda-\mu}-A(\lambda) A(\mu) \frac{\left(\gamma^{[k]}(\lambda)-\gamma^{[k]}(\mu)\right)}{\lambda-\mu}, \\
& \{D(\lambda), B(\mu)\}^{(k)}=-\frac{\mu^{k}(D(\lambda) B(\mu)-D(\mu) B(\lambda))}{\lambda-\mu}-\beta^{[k-1]} D(\lambda) A(\mu) \\
& \{D(\lambda), C(\mu)\}^{(k)}=\frac{\mu^{k}(D(\lambda) C(\mu)-D(\mu) C(\lambda))}{\lambda-\mu}+\gamma^{[k-1]} D(\lambda) A(\mu) \\
& \{B(\lambda), C(\mu)\}^{(k)}=-\frac{\mu^{k} A(\lambda) D(\mu)-\lambda^{k} A(\mu) D(\lambda)}{\lambda-\mu}
\end{aligned}
$$

and

$$
\begin{aligned}
\{A(\lambda), D(\mu)\}^{(k)}= & \frac{\lambda^{k}(C(\lambda) B(\mu)-C(\mu) B(\lambda))}{\lambda-\mu} \\
& -\frac{A(\lambda)}{\lambda-\mu}\left(B(\mu)\left(\beta^{[k]}(\lambda)-\beta^{[k]}(\mu)\right)-C(\mu)\left(\gamma^{[k]}(\lambda)-\gamma^{[k]}(\mu)\right)\right) .
\end{aligned}
$$

Here $\beta^{[k]}=\lambda^{k} B(\lambda) / A(\lambda)$ (3.11) and $\gamma^{[k]}=\lambda^{k} C(\lambda) / A(\lambda)$ are polynomial parts of the Laurent decompositions of quotients of the corresponding polynomials.

So, at $k>1$ we have to add to the matrices $r_{1,2}$ and $s$ dynamical terms proportional to the functions $\beta^{[k]}$ and $\gamma^{[k]}$.

According to $[7,19,20]$ the separated coordinates are poles of the corresponding BakerAkhiezer function with the standard normalization $\vec{\alpha}=(0,1)$. In this case the first half of variables are coming from $(n-1)$ finite roots and logarithm of leading coefficient of the nondiagonal entry of the monodromy matrix

$$
B(\lambda)=-e^{-v_{n}} \prod_{j=1}^{n-1}\left(\lambda-u_{j}\right), \quad v_{n}=-q_{n},
$$

Another half is given by

$$
v_{j}=-\ln A\left(u_{j}\right), \quad j=1, \ldots, n-1, \quad \text { and } \quad u_{n}=-c_{1}=\sum_{i=1}^{n} p_{i} .
$$

In the separated variables polynomial $A(\lambda)$ reads as

$$
A(\lambda)=\left(\lambda+\sum_{j=1}^{n} u_{j}\right) \prod_{j=1}^{n-1}\left(\lambda-u_{j}\right)+\sum_{j=1}^{n-1} e^{-v_{j}} \prod_{i \neq j}^{n-1} \frac{\lambda-u_{i}}{u_{j}-u_{i}}
$$

This definition of the separated variables is obviously related with the following transformation $\mathcal{X} \rightarrow \mathcal{X}^{-1}$ of the Weyl function [25].

It follows from (3.16) that at $k=0,1$

$$
\{A(\lambda), B(\mu)\}^{(k)}=\frac{1}{\lambda-\mu}\left(\mu^{k} A(\lambda) B(\mu)-\lambda^{k} A(\mu) B(\lambda)\right) .
$$

Substituting these brackets into the equations

$$
0=\left\{A(\lambda), B\left(u_{j}\right)\right\}^{(k)}=\left.\{A(\lambda), B(\mu)\}^{(k)}\right|_{\mu=u_{j}}+B^{\prime}\left(u_{j}\right)\left\{A(\lambda), u_{j}\right\}^{(k)}
$$


one gets

$$
\left\{A(\lambda), u_{j}\right\}^{(k)}=\lambda^{k} A\left(u_{j}\right) \prod_{i \neq j}^{n-1} \frac{\lambda-u_{i}}{u_{j}-u_{i}}, \quad j=1, \ldots, n-1 .
$$

If $\lambda=u_{i}$ this implies part of the necessary relation (2.14)

$$
\left\{u_{j}, v_{i}\right\}^{(k)}=u_{i}^{k} \delta_{i j}, \quad k=0,1, \quad i, j=1, \ldots, n-1 .
$$

Now we can collect all the coefficients with the same powers of $\lambda$ and $\mu$ in (3.20) and prove that

$$
\begin{array}{lll}
\left\{u_{n}, v_{n}\right\}=1, & \left\{u_{i}, u_{n}\right\}=0, & \left\{v_{n}, v_{i}\right\}=0, \\
\left\{u_{n}, v_{n}\right\}^{\prime}=-\sum_{i=1}^{n} u_{i}, & \left\{u_{n}, u_{i}\right\}^{\prime}=f_{i}=\frac{e^{-v_{i}}}{\prod_{j \neq i}^{n-1}\left(u_{i}-u_{j}\right)}, & \left\{v_{n}, v_{i}\right\}^{\prime}=-1 .
\end{array}
$$

In a similar manner from $\{B(\lambda), B(\mu)\}^{(k)}=0$ one gets

$$
\left\{u_{i}, u_{j}\right\}^{(k)}=\left\{v_{n}, u_{j}\right\}^{(k)}=0, \quad i, j=1, \ldots, n-1,
$$

and from $\{A(\lambda), A(\mu)\}^{(k)}=0$ it follows that

$$
\begin{array}{ll}
\left\{u_{n}, v_{i}\right\}=0, & \left\{v_{i}, v_{j}\right\}=0, \quad i, j=1, \ldots, n-1, \\
\left\{u_{n}, v_{i}\right\}^{\prime}=g_{i}=-\left.e^{-v_{n}} \frac{A^{\prime}(\lambda)}{B^{\prime}(\lambda)}\right|_{\lambda=u_{i}}, & \left\{v_{i}, v_{j}\right\}^{\prime}=0 .
\end{array}
$$

Here $A^{\prime}(\lambda)$ and $B^{\prime}(\lambda)$ are derivatives by $\lambda$.

Summing up, the $2 n$ separated variables $v_{i}$ and $u_{i}$ are the Darboux variables

$$
\omega=\sum_{i=1}^{n} d u_{i} \wedge d v_{i}
$$

but the corresponding recursion operator $N$ consists of two diagonal and three non-diagonal terms

$$
\begin{aligned}
N= & \sum_{i=1}^{n-1} u_{i}\left(\frac{\partial}{\partial u_{i}} \otimes d u_{i}+\frac{\partial}{\partial v_{i}} \otimes d v_{i}\right)-\sum_{i=1}^{n} u_{i}\left(\frac{\partial}{\partial u_{n}} \otimes d v_{n}+\frac{\partial}{\partial v_{n}} \otimes d u_{n}\right) \\
& +\sum_{i=1}^{n-1} f_{i}\left(\frac{\partial}{\partial u_{i}} \otimes d u_{n}-\frac{\partial}{\partial u_{n}} \otimes d u_{i}\right)+\sum_{i=1}^{n-1}\left(\frac{\partial}{\partial v_{n}} \otimes d v_{i}-\frac{\partial}{\partial v_{i}} \otimes d v_{n}\right) \\
& +\sum_{i=1}^{n-1} g_{i}\left(\frac{\partial}{\partial v_{i}} \otimes d u_{n}+\frac{\partial}{\partial u_{n}} \otimes d v_{i}\right) .
\end{aligned}
$$

Thus $2 n$ separated variables $v_{i}$ and $u_{i}$ obtained in framework of the Sklyanin method [19] are not the Darboux-Nijenhuis variables. Evidently it is related with the difference in the form of the corresponding separated equations, which follow directly from the definitions of $v_{j}$ and $u_{n}(3.18)$

$$
\Phi_{j}=e^{-v_{j}}-\Delta\left(u_{j}, \alpha_{1}, \ldots, \alpha_{n}\right)=0, \quad \Phi_{n}=u_{n}+\alpha_{1}=0 .
$$


The first $(n-1)$ equations of motion, see (3.21), are linearized by the Abel transformation [19, 21]

$$
\left\{A(\lambda), \sum_{k=1}^{n-1} \int^{v_{k}} \sigma_{j}\right\}=-\lambda^{j-1}, \quad \sigma_{j}=\frac{\lambda^{j-1} d \lambda}{\Delta_{N}(\lambda)}, \quad j=1, \ldots, n-1,
$$

where $\left\{\sigma_{j}\right\}$ is a basis of Abelian differentials of first order on an algebraic curve $z=\Delta_{N}(\lambda)$ corresponding to separated equations (3.23).

It means that we can introduce the action-angle variables

$$
I_{j}=c_{j}, \quad w_{1}=v_{n}, \quad \text { and } \quad w_{j+1}=\sum_{k=1}^{n-1} \int^{v_{k}} \sigma_{j},
$$

such that evolution of variables $w_{j}$ with respect to times $\tau_{j}$ conjugated to the action variables $I_{j}$ are linear. These action variables are related with the previous ones

$$
A(\lambda)=\prod_{i=1}^{n}\left(\lambda-\lambda_{i}\right)=\lambda^{n}-\sum_{j=1}^{n} I_{j} \lambda^{n-j} .
$$

Bi-Hamiltonian integrals of motion $H_{j}$ (1.6) produce the flows which preserve the spectrum of the $n \times n$ Jacobi matrix $L$ (3.3). Integrals of motion $I_{j}=c_{j}$ produce the transversal to the isospectral manifolds flows, which preserve the divisor [25].

As sequence the special Darboux-Nijenhuis variables $\lambda, \mu$ are dual to the Sklyanin variables $u, v$. Namely, $\lambda_{i}, \mu_{i}$ are roots of polynomial $A(\lambda)$ and values of polynomial $B(\lambda)$ at $\lambda=\lambda_{i}$, while $u_{j}, v_{j}$ are roots of polynomial $B(\lambda)$ and values of polynomial $A(\lambda)$ at $\lambda=u_{j}$.

Remark 2. From the factorization of the monodromy matrix $T(\lambda)(3.15)$ one gets

$$
B_{n}(\lambda)=-e^{q_{n}} A_{n-1}(\lambda) \quad \Rightarrow \quad B_{n}\left(u_{j}\right)=-e^{q_{n}} A_{n-1}\left(\lambda_{j}\right)=0 .
$$

This implies that the Moser variables $\lambda_{j}$ for a $(n-1)$-particle chain, i.e. special DarbouxNijenhuis variables, coincide with the Sklyanin variables $u_{j}, i=1, \ldots, n-1$ for a $n$-particle chain.

We can prove this fact directly using matrix representation of the recursion operator for $(n-1)$-particle chain, which can be obtained by the matrix representation (3.22) deleting the $n$-th and $2 n$-th rows and columns.

Remark 3. The Sklyanin variables are the separated variables for open and periodic Toda lattices simultaneously, in contrast with the Moser variables. For the periodic Toda lattice the Darboux-Nijenhuis variables were constructed by using Flaschka variables in [3].

Example 3. At $n=2$ the Sklyanin variables are equal to

$$
v_{1}=-\ln \left(-e^{q_{1}-q_{2}}\right), \quad v_{2}=-q_{2}, \quad u_{1}=p_{1}, \quad u_{2}=p_{1}+p_{2},
$$

and

$$
N=\left(\begin{array}{ccrc}
u_{1} & 0 & 0 & -e^{-v_{1}} \\
2 u_{1}-u_{2} & u_{2}-u_{1} & e^{-v_{1}} & 0 \\
0 & -1 & u_{1} & 2 u_{1}-u_{2} \\
1 & 0 & 0 & u_{2}-u_{1}
\end{array}\right)
$$

We have three different families of the separated variables for the Toda lattice at $n=2$ only. 


\section{Conclusion}

For the open Toda lattice associated with the root system of $\mathscr{A}_{n}$ type we prove that the Moser variables are special Darboux-Nijenhuis variables, while the Sklyanin variables are "almost Darboux-Nijenhuis variables", in which recursion operator consists of a diagonal part and two non-diagonal rows and columns only.

The similar results for the generalized open Toda lattices associated with the root systems of $\mathscr{B}_{n}, \mathscr{C}_{n}$ and $\mathscr{D}_{n}$ type were found in [24].

Nevertheless we have to underline that construction of the Moser variables and the Sklyanin variables directly in the framework of the bi-Hamiltonian geometry is an open question.

\section{Acknowledgements}

We would like to thank I.V. Komarov and V.I. Inozemtsev for useful and interesting discussions. The research was partially supported by the RFBR grant 06-01-00140.

[1] Atiyah M., Hitchin N., The geometry and dynamics of magnetic monopoles, M.B. Porter Lectures, Princeton Univ. Press, 1988.

[2] Das A., Okubo S., A systematic study of the Toda lattice, Ann. Phys., 1989, V.30, 215-232.

[3] Falqui G., Magri F., Pedroni M., Bihamiltonian geometry and separation of variables for Toda lattices, J. Nonlinear Math. Phys., 2001, V.8, 118-127, nlin.SI/0002008.

[4] Falqui G., Pedroni M., Separation of variables for bi-Hamiltonian systems, Math. Phys. Anal. Geom., 2003, V.6, 139-179, nlin.SI/0204029.

[5] Faybusovich L., Gekhtman M., Poisson brackets on rational functions and multi Hamiltonian structures for integrable lattices, Phys. Lett. A, 2000, V.272, 236-244, nlin.SI/0006045.

[6] Fernandes R.L., On the master symmetries and bi-Hamiltonian structure of the Toda lattice, J. Phys. A: Math. Gen., 1993, V.26, 3797-3803.

[7] Flaschka H., McLaughlin D.W., Canonically conjugate variables for the Korteweg-de Vries equation and the Toda lattice with periodic boundary conditions, Progr. Theoret. Phys., 1976, V.55, 438-456.

[8] Fokas A., Fuchssteiner B., Symplectic structures, Bäcklund transformations and hereditary symmetries, Phys. D, 1981, V.4, 47-66.

[9] Frölicher A., Nijenhuis A., Theory of vector-valued differential forms. I. Derivations of the graded ring of differential forms, Nederl. Akad. Wetensch. Proc. Ser. A, 1956, V.59, 338-359.

[10] Gelfand I.M., Dorfman I.Ya., Hamiltonian operators and algebraic structures associated with them, Funktsional. Anal. i Prilozhen., 1979, V.13, N 4, 13-30.

[11] Gelfand I.M., Zakharevich I., On the local geometry of a bi-Hamiltonian structure, in The Gelfand Mathematical Seminars 1990-1992, Editors L. Corwin et al., Boston, Birkhauser, 1993, 51-112.

[12] Krein M.G., Naimark M.A., The method of symmetric and Hermitian forms in the theory of the separation of the roots of algebraic equations, Linear and Multilinear Algebra, 1981, V.10, N 4, 265-308 (translated from the Russian by O. Boshko and J.L. Howland).

[13] Kosmann-Schwarzbach Y., Magri F., Poisson-Nijenhuis structures, Ann. Inst. Poincaré (Phys. Theor.), 1990, V.53, 35-81.

[14] Komarov I.V., Tsiganov A.V., Two particle quantum periodic Toda lattice, Vestnik Leningrad Univ., 1988, V.2, 69-71.

[15] Levi-Civita T., Integrazione delle equazione di Hamilton-Jacobi per separazione di variabili, Math. Ann., 1904, V.24, 383-397.

[16] Magri F., Geometry and soliton equations, in La Mécanique Analytique de Lagrange et son héritage, Atti Acc. Sci. Torino Suppl., 1990, V.24, 181-209.

[17] Magri F., Eight lectures on integrable systems, in Integrability of Nonlinear Systems (1996, Pondicherry), Lecture Notes in Phys., Vol. 495, Berlin, Springer, 1997, 256-296.

[18] Moser J., Finitely many mass points on the line under the influence of an exponential potential - an integrable system, in Dynamical Systems, Theory and Applications (1974, Rencontres, BattelleRes. Inst., Seattle, Wash.), Lecture Notes in Phys., Vol. 38, Berlin, Springer, 1975, 467-497. 
[19] Sklyanin E.K., The quantum Toda chain, in Nonlinear Equations in Classical and Quantum Field Theory (1983/1984, Meudon/Paris), Lecture Notes in Phys., Vol. 226, Berlin, Springer, 1985, 196-293.

[20] Sklyanin E.K., Separation of variables - new trends, in Quantum Field Theory, Integrable Models and Beyond (1994, Kyoto), Progr. Theoret. Phys. Suppl., 1995, V.118, 35-60, solv-int/9504001.

[21] Smirnov F.A., Structure of matrix elements in quantum Toda chain, J. Phys. A: Math. Gen., 1998, V.31, 8953-8971, math-ph/9805011.

[22] Stieltjes T., Recherches sur les fractions continues, Ann. de Toulouse, VIII-IX, 1894-1895.

[23] Tsiganov A.V., On the invariant separated variables, Regul. Chaotic Dyn., 2001, V.6, 307-326.

[24] Tsiganov A.V., On the Darboux-Nijenhuis coordinates for the generalizaed open Toda lattices, Theoret. and Math. Phys., submitted.

[25] Vaninsky K.L., The Atiyah-Hitchin bracket and the open Toda Lattice, J. Geom. Phys., 2003, V.46, 283307, math-ph/0202047. 